\title{
Tomographic Spectral Imaging: Analysis of Localized Corrosion
}

\author{
P.G. Kotula, M.R. Keenan, and J.R. Michael \\ Sandia National Laboratories, PO Box 5800, Albuquerque, NM 87185-0886
}

Microanalysis is typically performed to analyze the near surface of materials. There are many instances where chemical information about the third spatial dimension is essential to the solution of materials analyses. The majority of 3D analyses however focus on limited spectral acquisition and/or analysis. For truly comprehensive 3D chemical characterization, 4D spectral images (a complete spectrum from each volume element of a region of a specimen) are needed. Furthermore, a robust statistical method is needed to extract the maximum amount of chemical information from that extremely large amount of data [1].

In this paper, an example of the acquisition and multivariate statistical analysis of 4D (3-spatial and 1 -spectral dimension) $\mathrm{x}$-ray spectral images is described [2]. The method of utilizing a single- or dual-beam FIB (w/o or w/SEM) to get at 3D chemistry has been described by others with respect to secondary-ion mass spectrometry [3,4]. The basic methodology described in those works has been modified for comprehensive x-ray microanalysis in a dual-beam FIB/SEM (FEI Co. DB-235) [2]. In brief, the FIB is used to serially section a site-specific region of a sample and then the electron beam is rastered over the exposed surfaces with x-ray spectral images being acquired at each section. All this is performed without rotating or tilting the specimen between FIB cutting and SEM imaging/xray spectral image acquisition. The resultant $4 \mathrm{D}$ spectral image is then unfolded (number of volume elements by number of channels) and subjected to the same multivariate curve resolution (MCR) approach [2] that has proven successful for the analysis of lower-dimension X-ray spectral images. The TSI data sets can be in excess of 4Gbytes. This problem has been overcome (for now) and images up to 6Gbytes have been analyzed in this work. The method for analyzing such large spectral images will be described in this presentation. A comprehensive 3D chemical analysis was performed on several corrosion specimens of $\mathrm{Cu}$ electroplated with various metals.

Figure 1A shows the top view of the localized corrosion region prepared for FIB sectioning. The TSI region has been coated with $\mathrm{Pt}$ and a trench has been milled along the bottom edge of the region, exposing it to the electron beam as seen in Figure 1B. The TSI consisted of 25 sections and was approximately 6Gbytes.Figure $1 \mathrm{C}$ shows several of the components rendered in 3D: Green is $\mathrm{Cu}$; blue is $\mathrm{Pb}$; cyan represents one of the corrosion products that contains $\mathrm{Cu}, \mathrm{Zn}, \mathrm{O}, \mathrm{S}$, and $\mathrm{C}$; and orange represents the other corrosion product with $\mathrm{Zn}, \mathrm{O}, \mathrm{S}$ and $\mathrm{C}$. Figure $1 \mathrm{D}$ shows all of the component spectral shapes from the analysis. There is severe pathological overlap of the spectra from $\mathrm{Ni}, \mathrm{Cu}$ and $\mathrm{Zn}$ as well as $\mathrm{Pb}$ and $\mathrm{S}$. in spite of this clean spectral shapes have been extracted from the TSI. This powerful TSI technique could be applied to other sectioning methods well [5].

[1] P.G. Kotula, M.R. Keenan, and J.R. Michael, Microsc. Microanal. 9 (2003) 1-17.

[2] P.G. Kotula, M.R. Keenan, and J.R. Michael, Microsc. Microanal. 9 (Suppl. 2) (2003) 1004.

[3] T. Sakamoto et al., Jpn. J. Appl. Phys. 37 (1998) 2051-2056.

[4] D.N. Dunn and R. Hull, Appl. Phys. Lett. 75 (1999) 3414-3416.

[5] Sandia is a multiprogram laboratory operated by Sandia Corporation, a Lockheed Martin

Company, for the United Stated Department of Energy (DOE) under contract DE-AC0494AL85000. 


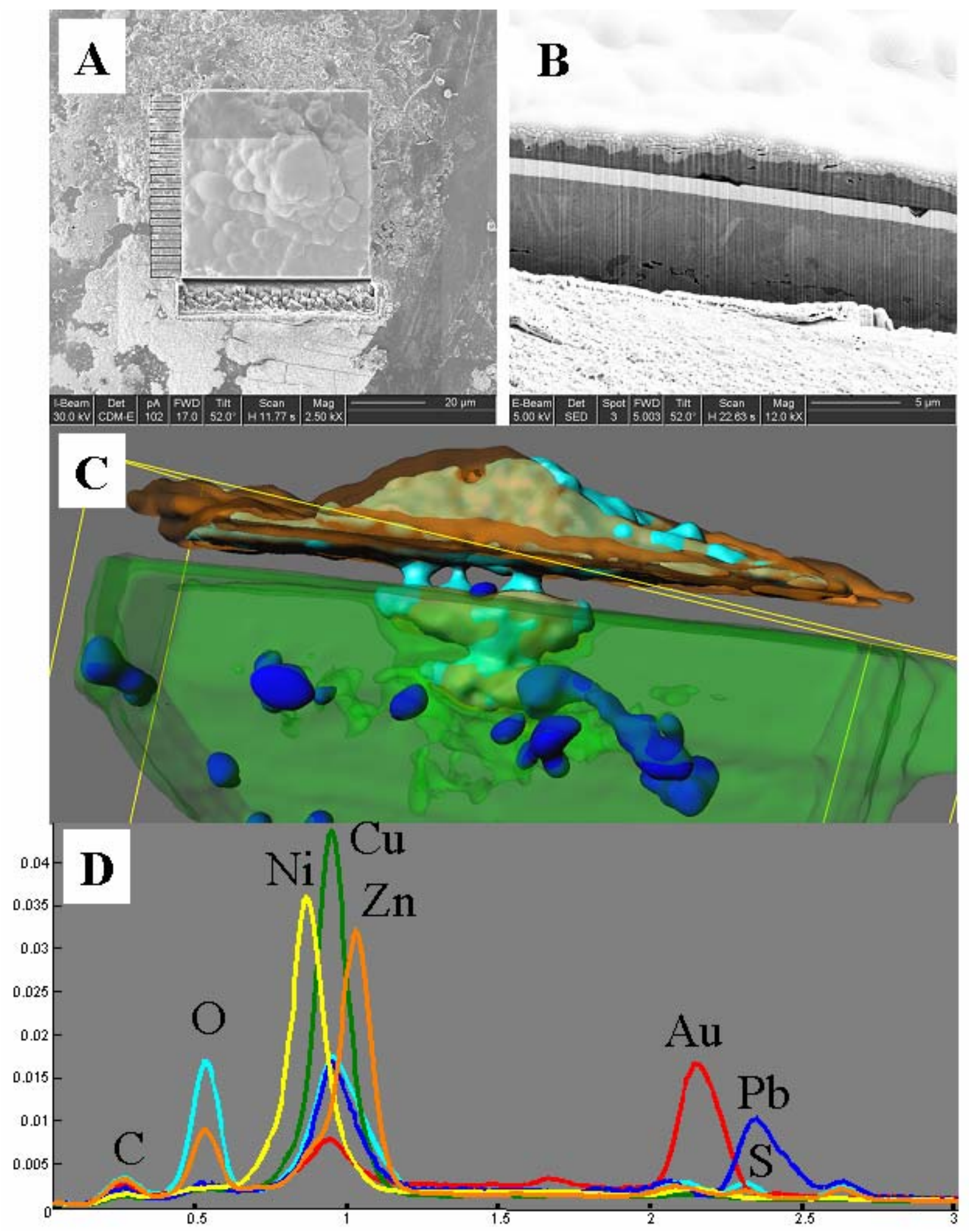

FIG. 1. A. Ion image of the site-specific region of the TSI. B. Electron image of one section. C. Some of the component images from the analysis of the TSI. D. All of the component spectral shapes from the analysis of the TSI. 